\title{
Effects of Spraying salicylic acid on Breaking Dormancy and Antioxidant Substances of Ziziphus jujuba 'Zhanshanmizao'
}

\author{
Shangyu Tao ${ }^{1, a}$, Jie Zhou ${ }^{1, b}$, Yan Zhou ${ }^{1, \mathrm{c}}$ and Huifen Zhang ${ }^{1, \mathrm{~d} *}$ \\ ${ }^{1}$ College of Horticulture, Sichuan Agricultural University, Chengdu, Sichuan, China \\ a2693245395@qq.com, b493137598@qq.com, 'zhouyan6213500@qq.com, \\ d1042335395@qq.com
}

${ }^{*}$ Corresponding author.

Keywords: SA; Ziziphus jujuba 'Zhanshanmizao'; enforced dormancy; antioxidant system Abstract. Seven-year-old tree of Ziziphus jujuba 'Zhanshanmizao' cultivated in field were used to study the breaking dormancy and the dynamic changes of antioxidants (superoxide dismutase, peroxidase, $\mathrm{O}_{2} \bullet$, and malonyldialdehyde $)$ in jujube shoots under different concentrations $(2 \mathrm{mmol} / \mathrm{L}$, $4 \mathrm{mmol} / \mathrm{L}$, and $6 \mathrm{mmol} / \mathrm{L}$ ) of salicylic acid (SA). The results showed that $6 \mathrm{mmol} / \mathrm{L}$ SA could significantly activate the antioxidant system in the $Z$. jujuba 'Zhanshanmizao' buds during the enforced dormancy period (January 30 th to February 14 th). This concentration of SA is better than other concentration to break the enforced dormancy of the Z. jujuba 'Zhanshanmizao' bud. $2 \mathrm{mmol} / \mathrm{L}$ SA had little effect on breaking the dormancy of Z. jujuba 'Zhanshanmizao'.

\section{Introduction}

Ziziphus jujuba 'Zhanshanmizao' is a deciduous fruit tree. It has a long history of cultivation, and the jujube fruit is rich in a variety of therapeutic and health substances. It is an excellent fresh spot with local characteristics that is well received by the market [1]. In recent years, relatively more studies have been done to replace the dormancy of deciduous fruit trees with chemical agents instead of cryogenic temperatures [2]. But so far there have been few reports on breaking the physiology of enforced dormancy of Z. jujuba 'Zhanshanmizao'. Salicylic acid (SA) is currently used in many studies of dormancy in deciduous fruit trees. In this study, seven-year-old tree of Z. jujuba 'Zhanshanmizao' was used as material to study the dynamic changes of antioxidant substances in the shoots of Z. jujuba 'Zhanshanmizao' with different concentrations of SA. The aim is to filter out the most effective concentration of SA that breaks the enforced dormancy of Z. jujuba 'Zhanshanmizao'. Then advance the mature period of $Z$. jujuba 'Zhanshanmizao', increase the income of the juvenile farmers and this will provide some guidance for the regulation of the ripening period of $Z$. jujuba 'Zhanshanmizao'.

\section{Materials and Methods}

Materials. The tested material was 7 years old jujube (tillering propagation seedling), spaced at 2 $\mathrm{m} \times 3 \mathrm{~m}$. Orchard is located in Yongxin Zhen Yonglian Village, Santai County, Mianyang City. Orchard for hilly terraces, yellow red purple soil.

The plants were processed on January 30 th, 2017. Set 4 levels and compared with fresh water treatment. In the individual plots, each treatment was repeated 3 times for a total of 36 strains, arranged in random blocks. The first sampling was performed on the second day of treatment, and samples were taken every seven days. At each sampling time, two or three representative secondary branches were cut from the middle and upper parts of the perimeter of the jujube tree. After being wrapped in plastic wrap, the ice box was brought back to the Comprehensive Laboratory of Horticulture Department, Sichuan Agricultural University. The shoot with buds was removed with a blade and placed in a $-10^{\circ} \mathrm{C}$ refrigerator for analysis to analyze the dynamic changes of antioxidant substances. 
Methods. Superoxide dismutase (SOD) was measured using the method of nitrogen blue tetrazolium (NBT) method [3]. The determination of peroxidase (POD) was performed using guaiacol method [3]. The content of $\mathrm{O}_{2}-\bullet$ was determined with reference to $\mathrm{Bi}$ Lei [4]. The determination of malonyldialdehyde (MDA) content was based on Bi Lei [5].

\section{Results and Discussion}

Changes of SOD activity. SOD is an important enzyme of the antioxidant system in plants [6]. SA treatment greatly promoted the increase of SOD activity (Fig.1), and the SOD activity showed a "gradually increasing" trend, which was consistent with the results of Chen's research in apricot shoot buds [7]. The increase in SOD activity helps clear free radicals accumulated by the tree under low temperature stress and breaks the enforced dormancy. On the 15 th day, the SOD activity in the branches treated with $2 \mathrm{mmol} / \mathrm{L} \mathrm{SA}$ and $6 \mathrm{mmol} / \mathrm{L} \mathrm{SA}$ was the highest, indicating that the two treatments had stronger ability to activate the antioxidant system.

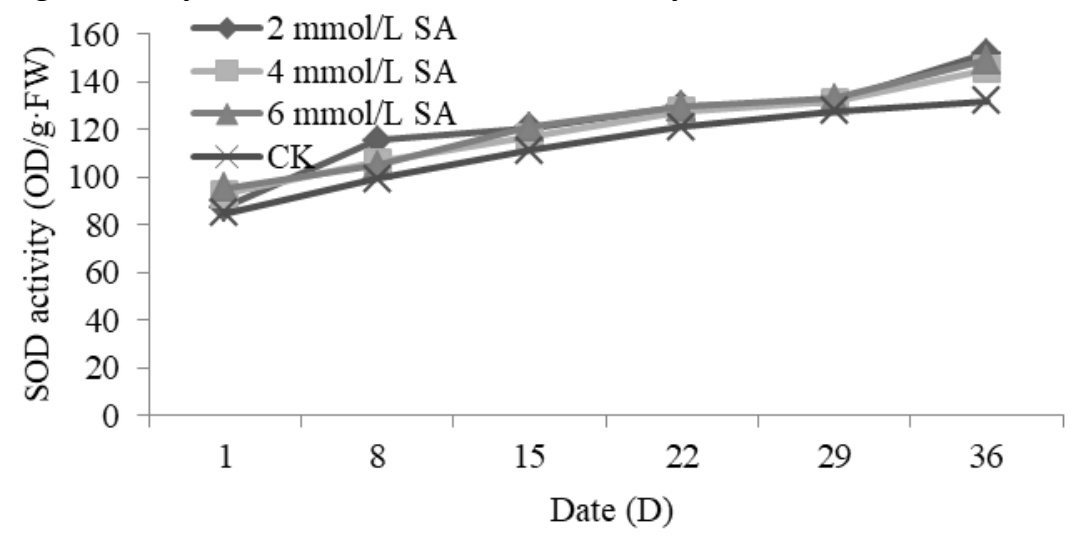

Fig.1 Changes of SOD activity

Changes of POD activity. Like SOD, POD is also an enzyme that protects against reactive oxygen species damage in plants [6]. The POD activity in the Z. jujuba 'Zhanshanmizao' buds first decreases and then rises (Fig.2). After spraying SA, POD activity was consistently higher during the whole enforced dormancy than that in the controls. After 15 days of treatment, the POD activity gradually increased, which may be related to the germination of the Z. jujuba 'Zhanshanmizao' buds. When the POD activity was at the bottom, the enforced dormancy was broken. In all treatments, the POD activity of the $6 \mathrm{mmol} / \mathrm{L} \mathrm{SA}$ treated shoots has the fastest change, which was significantly higher than that of other treatments.

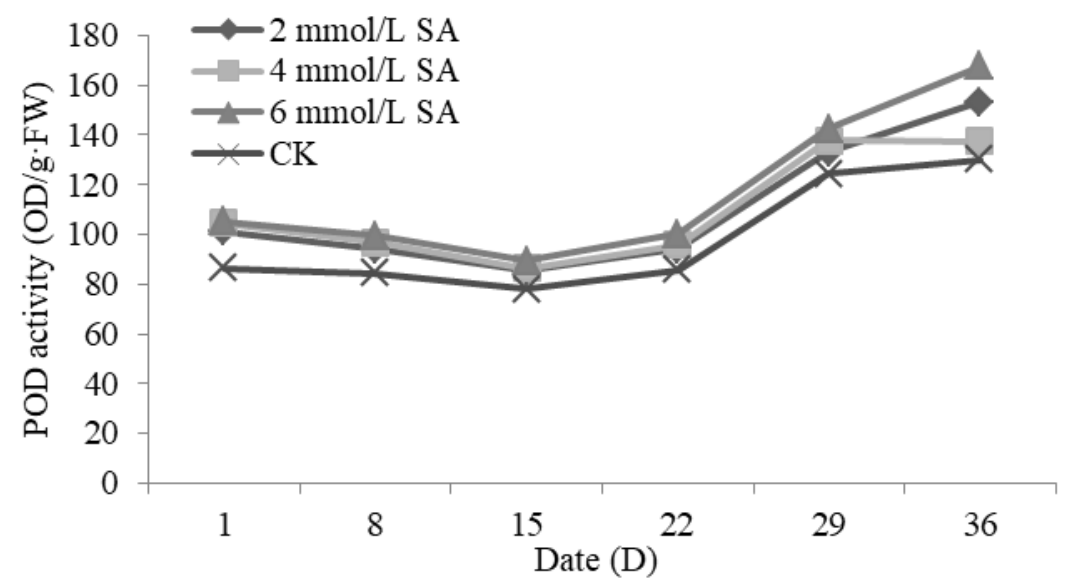

Fig.2 Changes of POD activity

Changes of $\mathrm{O}_{2}-\bullet$ content. The content of $\mathrm{O}_{2} \cdot \bullet$ in buds of $Z$. jujuba 'Zhanshanmizao' treated with different concentrations of SA was significantly lower than that of the control. The trend of "gradual decline" (Fig.3) is opposite to the change in SOD activity. In all the treatments, the content of $\mathrm{O}_{2}{ }^{-\bullet}$ in 
the Z. jujuba 'Zhanshanmizao' shoots sprayed with $6 \mathrm{mmol} / \mathrm{L} \mathrm{SA}$ was faster than that of other shoots with SA.

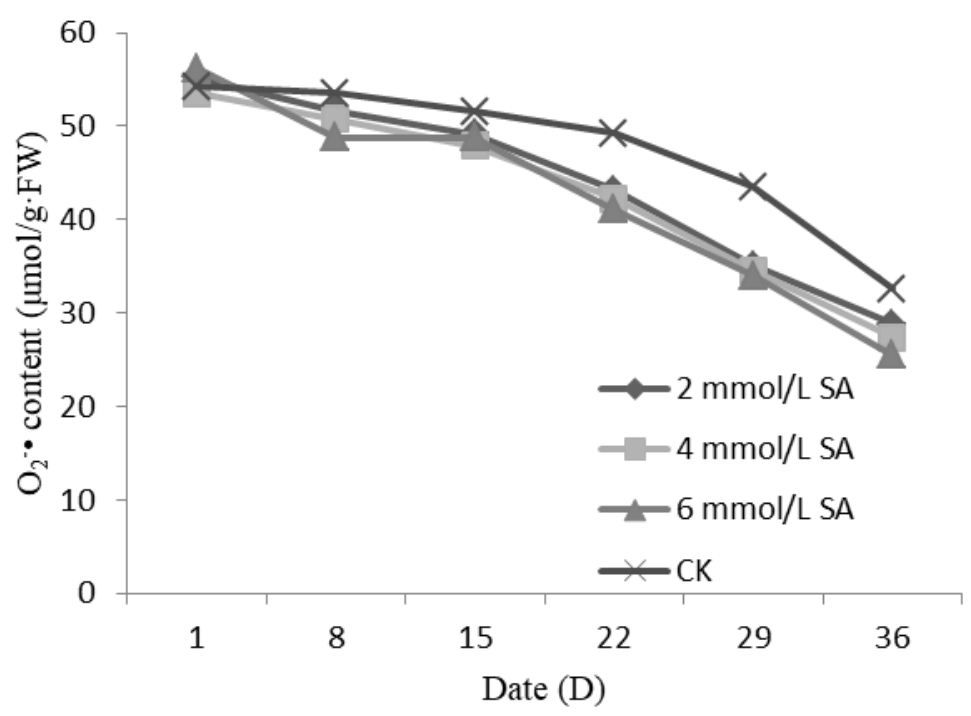

Fig. 3 Changes of $\mathrm{O}_{2} \bullet$ content

Changes of MDA content. Changes in membrane permeability are one of the dormant phenomena, and MDA is the final breakdown product of membrane lipid peroxidation. Its content can reflect the degree of lipid peroxidation [8]. After analysis of the data, the MDA content showed the trend of "change first and then change smoothly" (Fig.4). The trough values of all treatments appeared at the 15 th day of treatment. On the 15 th day, the content of MDA in shoots of Z. jujuba 'Zhanshanmizao' that was sprayed with $6 \mathrm{mmol} / \mathrm{L} \mathrm{SA}$ was higher than other treatments, but the content of MDA after 15 th days was lower than other treatments.

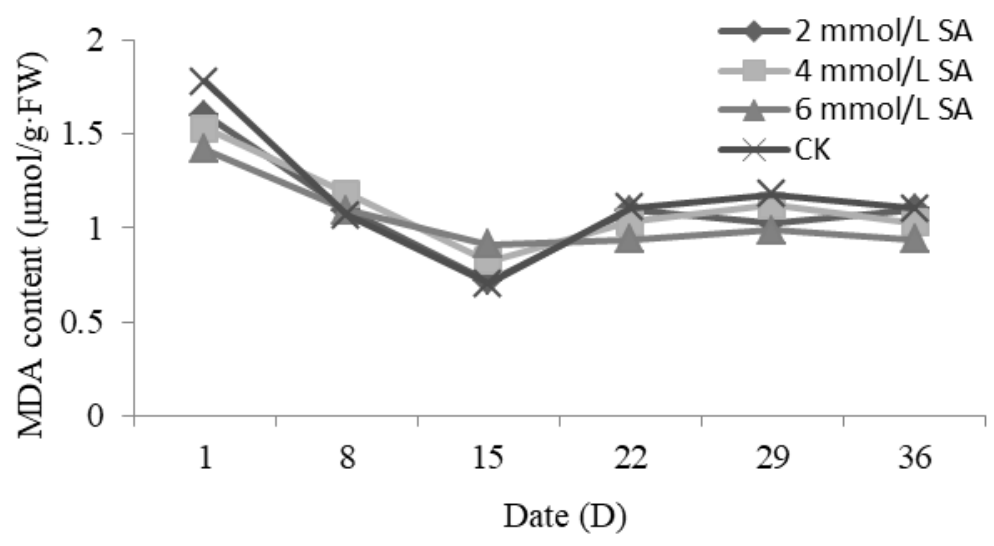

Fig.4 Changes of MDA content

\section{Discussion and conclusions}

Deciduous fruit tree dormancy is divided into related dormancy, endo-dormancy, and enforced dormancy [9]. Enforced dormancy is the satisfaction of internal factors such as coldness, but environmental stress (such as water stress, nutrient deficit, etc.) can not sprout. In the early stage of enforced dormancy, SOD activity was low due to inducing enzymes. With the changes of the outside temperature, the SOD activity gradually increased and the POD activity gradually decreased. After the enforced dormancy was broken, the POD activity gradually increased when the $Z$. jujuba 'Zhanshanmizao' shoots were ready to sprout. SOD is the first enzyme in the degradation of superoxide radicals. And its rise can be seen as an adaptive response to cold stress. The higher the SOD activity, the stronger the decomposing effect of participating in the low temperature, and the content of $\mathrm{O}_{2}-\bullet$ will gradually decrease. The POD activity rapidly decreased at the beginning of enforced dormancy, and then gradually increased after breaking dormancy. This is consistent with 
Pang's study that enzyme activity of antioxidant enzymes in buds during apricot dormancy [10]. The results of this experiment showed that $6 \mathrm{mmol} / \mathrm{L} \mathrm{SA}$ had the strongest ability to activate the enzyme activity of antioxidant system during the enforced dormancy of Z. jujuba 'Zhanshanmizao'.

Plant organs are harmed under adverse conditions, and membrane lipid peroxidation often occurs. MDA is one of the main products of membrane lipid peroxidation, and its content can reflect the degree of membrane lipid peroxidation [8]. In the later stage of treatment, the content of MDA in the shoots treated with $6 \mathrm{mmol} / \mathrm{L} \mathrm{SA}$ was the lowest, indicating that the effect of decapitation was better.

\section{Acknowledgements}

This work was financially supported by the double subject construction plan of Sichuan Agricultural University and special action plan project of science and technology rich people and strong county of Sichuan Province "Integration and demonstration of key technologies for industrialized development of Z. jujuba 'Zhanshangmizao' of Santai County”.

\section{References}

[1] L. Wang, A.M. Min, Z.G. Qin, Y. Wang, C.G. Li and H.X. Li: Journal of West China Forestry Science Vol.5 (2014), p. 48.

[2] P.Q. Sun, Y. Liu, J.M. Jia, X.H. Wang, Z.A. Shi and X.L. Li: Chinese Agricultural Science Bulletin Vol. 27 (2011), p. 222.

[3] Q.E. Xiong: An Experimental course in Plant Physiology (Sichuan Science and Technology Press, Chengdu 2003).

[4] L. Bi: Study on Changes of Inclusions and Metabolism during Dormant Period of Qiyuesu Pear (thesis of master degree, Agricultural University of Hebei, Baoding, China 2009).

[5] L. Bi: Studies on Dormant Characteristic and Releases from Dormancy of Pear (thesis of master degree, Agricultural University of Hebei, Baoding, China 2006).

[6] T.H. Zhou: Chinese Agricultural Science Bulletin Vol. 19 (2012), p. 141.

[7] D.W. Chen, F. Wang, A.Q. Gao and J.R. Li: Acta Botanica Boreali-Occidentalia Sinica Vol. 20 (2000), p. 212.

[8] D.M. Li, H.S. Zhang, Q.P. Tan, D.S. Gao, X.D. Chen and L. Li: Chinese Journal of Applied Ecology Vol. 27 (2016), p. 1837.

[9] L.H. Fuchigami and C.C. Nee: Hortscience Vol. 22 (1987), p. 836.

[10] F.H. Pang, J.J. Du and F. Liu: Journal of Hubei Institute for Nationalities (Natural Science Edition) Vol. 22 (2004), p. 27. 